EUROPEAN JOURNAL OF PURE AND APPLIED MATHEMATICS

Vol. 12, No. 2, 2019, 571-576

ISSN 1307-5543 - www.ejpam.com

Published by New York Business Global

\title{
Finite Groups With Certain Permutability Criteria
}

\author{
Rola A. Hijazi ${ }^{1}$, Fatme M. Charaf ${ }^{1, *}$ \\ ${ }^{1}$ Department of Mathematics, Faculty of Science, King Abdulaziz University, Jeddah, \\ Saudi Arabia
}

\begin{abstract}
Let $G$ be a finite group. A subgroup $H$ of $G$ is said to be $S$-permutable in $G$ if it permutes with all Sylow subgroups of $G$. In this note we prove that if $P$, the Sylow $p$-subgroup of $G(p>2)$, has a subgroup $D$ such that $1<|D|<|P|$ and all subgroups $H$ of $P$ with $|H|=|D|$ are $S$-permutable in $G$, then $G^{\prime}$ is $p$-nilpotent.
\end{abstract}

2010 Mathematics Subject Classifications: 20D10, 20D20

Key Words and Phrases: $S$-Permutable Subgroup, $p$-Nilpotent Group, Solvable Group, Supersolvable Group.

\section{Introduction}

Throughout this note, $G$ denotes a finite group. The relationship between the properties of the Sylow subgroups of a group $G$ and its structure has been investigated by many authors. Starting from Gaschütz and Itő ([10], Satz 5.7, p.436) who proved that a group $G$ is solvable if all its minimal subgroups are normal. In 1970, Buckely [4] proved that a group of odd order is supersolvable if all its minimal subgroups are normal (a subgroup of prime order is called a minimal subgroup). Recall that a subgroup is said to be $S$-permutable in $G$ if it permutes with all Sylow subgroup of $G$. This concept, as a generalization of normality, was introduced by Kegel [11] in 1962 and has been studied extensively in many notes. For example, Srinivasan [15] in 1980 obtained the supersolvability of $G$ under the assumption that the maximal subgroups of all Sylow subgroups are $S$-permutable in $G$. In 2000, Ballester-Bolinches et al. [3] introduced the $c$-supplementation concept of a finite group: A subgroup $H$ of a group $G$ is said to be $c$-supplemented in $G$ if there exists a subgroup $K$ of $G$ such that $G=H K$ and $H \cap K \leq H_{G}$, where $H_{G}=\operatorname{Core}_{G}(H)$ is the largest normal subgroup of $G$ contained in $H$. By using this concept they were able to prove that a group $G$ is solvable if and only if every Sylow subgroup of $G$ is $c$-supplemented in $G$. Moreover, as an application, they got the supersolvability of a group $G$ if all its minimal subgroups and the cyclic subgroups of order 4 are $c$-supplemented in $G$.

*Corresponding author.

DOI: https://doi.org/10.29020/nybg.ejpam.v12i2.3399

Email addresses: Rhijazi@kau.edu.sa (R. Hijazi), fa-sharaf@hotmail.com (F. Charaf) 
In 2014, Heliel [8] proved that $G$ is solvable if each subgroup of prime odd order of $G$ is $c$-supplemented in $G$. Also he proved that $G$ is solvable if and only if every Sylow subgroup of odd order of $G$ is $c$-supplemented in $G$. This improved and generalized the results of Hall [6, 7], Ballester-Bolinches and Guo [2], and Ballester-Bolinches et al. [3]. Heliel also posted the following conjecture:

Let $G$ be a finite group such that every non-cyclic Sylow subgroup $P$ of odd order of $G$ has a subgroup $D$ such that $1<|D| \leq|P|$ and all subgroups $H$ of $P$ with $|H|=|D|$ are c-supplemented in $G$. Is $G$ solvable?

In the same year, Li et al. [12] presented a counterexample to show that the answer of this conjecture is negative in general and then gave a generalization of Heliel's theorems.

Example 1. Let $G=A_{5} \times H$, where $A_{5}$ is the alternating group of degree 5 and $H$ is an elementary group of order $p^{n}$ with $p>5$ and $n \geq 2$. Then $G$ satisfies the condition of the preceding conjecture, but $G$ is not solvable.

In 2015, Hijazi [9] continued the above mentioned investigations and proved the following: Suppose that each Sylow subgroup $P$ of $G$ has a subgroup $D$ such that $1<|D|<|P|$ and all subgroups $H$ of $P$ with $|H|=|D|$ are $S$-permutable in $G$. Then $G$ is solvable.

The main goal of this note is to prove the following main theorem:

Main Theorem 1. Let $P$ be a Sylow p-subgroup of $G(p>2)$. Suppose that $P$ has a subgroup $D$ such that $1<|D|<|P|$ and all subgroups $H$ of $P$ with $|H|=|D|$ are $S$-permutable in $G$. Then $G^{\prime}$ is p-nilpotent.

As immediate consequences of the main theorem we have:

Corollary 1. Let $P$ be a Sylow p-subgroup of $G(p>2)$. Suppose that $P$ has a subgroup $D$ such that $1<|D|<|P|$ and all subgroups $H$ of $P$ with $|H|=|D|$ are permutable in $G$. Then $G^{\prime}$ is p-nilpotent.

Corollary 2 ([9], Theorem 3.1). Suppose that each Sylow subgroup $P$ of $G$ has a subgroup $D$ such that $1<|D|<|P|$ and all subgroups $H$ of $P$ with $|H|=|D|$ are S-permutable in $G$. Then $G$ is solvable.

Corollary 3 (Gaschütz and Itő [10], Satz 5.7, p.436 ). A group $G$ is solvable if all its minimal subgroups are normal.

\section{Proofs}

We first prove the following theorems:

Theorem 2. Let $P$ be a Sylow p-subgroup of a group $G$, where $p$ is an odd prime. If each subgroup of $P$ of order $p$ is $S$-permutable in $G$, then $G^{\prime}$ is p-nilpotent.

Proof. We prove the theorem by induction on $|G|$. Hence if each subgroup of $P$ of order $p$ is normal in $G$, then each subgroup of $G^{\prime}$ of order $p$ is normal in $G^{\prime}$. Let $L$ be a 
subgroup of $G^{\prime}$ such that $|L|=p$. Then $G / C_{G}(L) \subseteq A u t(L)$ and, since $A u t(L)$ is cyclic of order $p-1$, we have $G / C_{G}(L)$ is abelian. Thus $G^{\prime} \leq C_{G}(L)$ and so $L \leq Z\left(G^{\prime}\right)$. By ([10], Satz 5.5(a), p. 435), $G^{\prime}$ is $p$-nilpotent. Thus we may assume that there exists a subgroup $H$ of $P$ of order $p$ such that $H$ is not normal in $G$. By the hypothesis, $H$ is $S$-permutable in $G$ and hence by ([13], Lemma A), $O^{p}(G) \leq N_{G}(H)<G$. Let $M$ be a maximal subgroup of $G$ such that $N_{G}(H) \leq M<G$. Then $M \triangleleft G$ and $|G / M|=p$. By induction on $|G|, M^{\prime}$ is $p$-nilpotent. Hence if $O_{p^{\prime}}(G) \neq 1, G / O_{p^{\prime}}(G)$ satisfies the hypothesis of the theorem and so $\left(G / O_{p^{\prime}}(G)\right)^{\prime}=G^{\prime} O_{p^{\prime}}(G) / O_{p^{\prime}}(G) \cong G^{\prime} /\left(G^{\prime} \cap O_{p^{\prime}}(G)\right)$ is $p$-nilpotent which implies that $G^{\prime}$ is $p$-nilpotent. Thus assume that $O_{p^{\prime}}(G)=1$. Since $M^{\prime}$ char $M$ and $M \triangleleft G$, we have $M^{\prime} \triangleleft G$. As $M^{\prime}$ is $p$-nilpotent and $O_{p^{\prime}}(G)=1$, we have $M^{\prime}$ is a $p$-group. Then $P_{1} \triangleleft M$ where $P_{1}$ is a Sylow $p$-subgroup of $M$. By Schur-Zassenhaus Theorem [5, Theorem 6.2.1, p. 221], $M=P_{1} K$, where $K$ is a $p^{\prime}$-Hall subgroup of $M$. Hence if $C_{G}\left(P_{1}\right) \leq P_{1}, K$ is a $p^{\prime}$-group of automorphisms of $P_{1}$, and since $K$ leaves each subgroup of $P_{1}$ invariant because every subgroup of $P$ of prime order is $S$-permutable, then by ([14], Lemma 2.20), $K$ is cyclic. Let $Q$ be a Sylow $q$-subgroup of $K$, where $q$ is a prime divisor of the order of $K$. Hence if $p<q$, then $P_{1} Q=P_{1} \times Q$ and this means that $Q \leq C_{G}\left(P_{1}\right)$, a contradiction. Thus $p$ is the largest prime dividing $|G|$ and since $K$ is cyclic, it follows, by Burnside's $p$-Nilpotent Theorem ([10], Satz 2.8, p.420), that $P \triangleleft G$. But $G / P \cong K$, therefore $G / P$ is cyclic and so abelian, then $G^{\prime} \leq P$. This completes the proof of the theorem.

As a corollary of Theorem 2.1:

Corollary 4. If each subgroup of prime order of $G$ is $S$-permutable in $G$, then $G$ is solvable, $S \triangleleft G^{\prime}$ and $G^{\prime} / S$ is nilpotent, where $S$ is a Sylow 2-subgroup of $G^{\prime}$.

Proof. By Theorem 2.1, $G^{\prime}$ is $p$-nilpotent for each odd prime $p$ dividing $|G|$. So $G^{\prime} / S$ is nilpotent, $S$ is a Sylow 2-subgroup of $G^{\prime}$ and hence $G$ is solvable.

Theorem 3. Let $p$ be an odd prime and let $P$ be a Sylow p-subgroup of $G$. Suppose that $P$ has a subgroup $D$ such that $1<|D|<|P|$ and all subgroups $H$ of $P$ with $|H|=|D|$ are normal in $G$. Then $G^{\prime}$ is p-nilpotent.

Proof. We prove the theorem by induction on $|G|$. Clearly, $P \cap G^{\prime}$ is a Sylow $p$-subgroup of $G^{\prime}$. Set $P_{1}=P \cap G^{\prime}$. We deal with the following two cases:

Case 1. $\left|P_{1}\right| \leq|D|$.

Hence if $|D|=p,\left|P_{1}\right|=p$, and $P_{1} \triangleleft G$. Then $G^{\prime} \leq C_{G}\left(P_{1}\right)$ and so $P_{1} \leq Z\left(G^{\prime}\right)$. Hence, by Schur-Zassenhaus Theorem, $G^{\prime}=P_{1} \times K$, where $K$ is a $p^{\prime}$-Hall subgroup of $G^{\prime}$.

In particular, $G^{\prime}$ is $p$-nilpotent.

Thus we may assume that $|D|=p^{n}(n \geq 2)$. Let $H$ be a subgroup of $P$ with $|H|=|D|$ such that $P_{1} \leq H<P$. By the hypothesis, $H \triangleleft G$. Assume that $\Phi(H) \neq 1$ and consider the factor group $G / \Phi(H)$. Obviously, $G / \Phi(H)$ satisfies the theorem hypothesis and so $(G / \Phi(H)) \prime=G^{\prime} \Phi(H) / \Phi(H)$ is $p$-nilpotent by the induction on $|G|$. But $G^{\prime} \Phi(H) / \Phi(H) \cong$ $G^{\prime} / G^{\prime} \cap \Phi(H)$ and $\Phi(H) \leq \Phi(G)$, then we have $G^{\prime} \cap \Phi(H) \leq G^{\prime} \cap \Phi(G)$ and therefore 
$G^{\prime} / G^{\prime} \cap \Phi(G)$ is p-nilpotent. Now $G^{\prime} \Phi(G) / \Phi(G) \cong G^{\prime} / G^{\prime} \cap \Phi(G)$ is $p$-nilpotent implies that $G^{\prime} \Phi(G)$ is p-nilpotent and consequently $G^{\prime}$ is $p$-nilpotent.

Thus we may assume that $\Phi(H)=1$ and so $H$ is elementary abelian $p$-group of order $p^{n}(n \geq 2)$. Let $L$ be a subgroup of $P$ contains $H$ such that $H$ is maximal in $L$. Clearly, $L$ is not cyclic because $H$ is elementary abelian group of order $p^{n}(n \geq 2)$. Then $L$ contains a subgroup $H_{1}$ such that $\left|H_{1}\right|=|D|$ and $H_{1} \neq H$. By the hypothesis, $H_{1} \triangleleft G$ and since $H \triangleleft G$, we have $L=H_{1} H \triangleleft G$ and so $\Phi(L) \leq \Phi(G)$. Hence if $\Phi(L) \neq 1, \Phi(L) \leq H_{1}<L \leq P$. Since $L$ is not cyclic, we have $\Phi(L)$ is contained properly in $H_{1}$. Now it is easy to notice that the factor group $G / \Phi(L)$ satisfies the hypothesis of the theorem, so by induction on $|G|, G^{\prime}$ is $p$-nilpotent. Thus we may assume that $\Phi(L)=1$ and so $P_{1}$ is elementary abelian $p$-group. Since $P_{1} \leq H<L \leq P$ and $H$ is maximal in $L$, it follows that $|L|=p^{n+1}$. Let $L_{1}=<x_{1}>$ be a subgroup of $P_{1}$ of order $p$. Then $L=<x_{1}>\times<x_{2}>\times \ldots \times<x_{n+1}>$. By the hypothesis, each maximal subgroup of $L$ is normal in $G$. Applying ([1], Lemma 2.9 ) implies that each subgroup of $L$ of order $p$ is normal in $G$; in particular each subgroup $L_{1}$ of $P_{1}$ of order $p$ is normal in $G$. So, $G^{\prime} \leq C_{G}\left(L_{1}\right)$ and consequently $P_{1} \leq Z\left(G^{\prime}\right)$. By Schur-Zassenhaus Theorem, $G^{\prime}=P_{1} \times K_{1}$, where $K_{1}$ is a $p^{\prime}$-Hall subgroup of $G$; in particular $G^{\prime}$ is $p$-nilpotent.

Case 2. $\left|P_{1}\right|>|D|$.

Hence if $|D|=p$, then every subgroup of $P_{1}$ of order $p$ is normal in $G$, so $\Omega_{1}\left(P_{1}\right) \leq$ $Z\left(G^{\prime}\right)$ which implies that $G^{\prime}$ is $p$-nilpotent by ([10], Satz 5.5(a), p 435). Thus assume that $|D|=p^{n}(n \geq 2)$. Hence if $\Phi(D) \neq 1, G / \Phi(D)$ satisfies the hypothesis of the theorem and so $(G / \Phi(D)) \prime=G^{\prime} \Phi(D) / \Phi(D)$ is p-nilpotent by induction on $|G|$ which implies that $G^{\prime} / G^{\prime} \cap \Phi(G)$ is $p$-nilpotent; in particular $G^{\prime}$ is $p$-nilpotent. Thus we may assume that $\Phi(D)=1$. Let $L \leq P_{1}$ such that $D$ is maximal in $L$. Then $|L|=p^{n+1}(n \geqslant 2)$. Clearly $L$ is not cyclic. Then there exists a maximal subgroup $L_{1} \neq D$ in $L$. By the hypothesis $L_{1} \triangleleft G$ and $D \triangleleft G$ which implies that $L=L_{1} D \triangleleft G$. Hence if $\Phi(L) \neq 1$, $\Phi(L) \leq D<L \leq P_{1}$ and since $L$ is not cyclic, it follows that $\Phi(L)<D$. By induction on $|G|, G^{\prime} \Phi(L) / \Phi(L) \cong G^{\prime} / G^{\prime} \cap \Phi(L)$ is p-nilpotent. In particular, $G^{\prime} \Phi(G) / \Phi(G)$ is $p$ nilpotent and it follows easily that $G^{\prime}$ is $p$-nilpotent. So we may assume that $\Phi(L)=1$ and so $L$ is elementary abelian. Let $L_{1}<P$ such that $\left|L_{1}\right|=p$. Then $L_{1}<L \leq P_{1}$ and so $L_{1} \triangleleft G$ by ([1], Lemma 2.9). In particular, $\Omega_{1}\left(P_{1}\right) \leq Z\left(G^{\prime}\right)$. Again by ([10], Satz $5.5(\mathrm{a}), \mathrm{p} 435), G^{\prime}$ is $p$-nilpotent. This completes the proof of the theorem.

Now we can move forward to prove our main theorem:

Proof. We prove the theorem by induction on $|G|$. Hence if $O_{p^{\prime}}(G) \neq 1, G / O_{p^{\prime}}(G)$ satisfies the hypothesis of the theorem and so $\left(G / O_{p^{\prime}}(G)\right) \prime$ is $p$-nilpotent by induction on $|G|$; in particular, $G^{\prime}$ is $p$-nilpotent. Thus we may assume that $O_{p^{\prime}}(G)=1$. If each subgroup $H$ of $P$ with $|H|=|D|$ is normal in $G$, then $G^{\prime}$ is $p$-nilpotent by Theorem 2.2. So we may assume that there exists a subgroup $H$ of $P$ with $|H|=|D|$ and $H$ is not normal in $G$. By hypothesis, $H$ is $S$-permutable in $G$. Since $H \Varangle G$ and $H$ is $S$-permutable in $G$, we have by ([13], Lemma A) that $O^{p}(G) \leq N_{G}(H)<G$. Let $M$ be a maximal subgroup of $G$ contains $N_{G}(H)$ properly. Then $M \triangleleft G$ and $|G / M|=p$. Let $P_{1}=P \cap M$ be a Sylow $p$-subgroup of $M$. By the hypothesis, $|D| \leq\left|P_{1}\right|$. If $|D|=\left|P_{1}\right|$, then $|H|=\left|P_{1}\right|$ and so 
$P \leq N_{G}(H)$, and since $O^{p}(G) \leq N_{G}(H)$, we have $P O^{p}(G)=G \leq N_{G}(H)<M$ which is impossible. Thus we may assume that $|D|<\left|P_{1}\right|$. Now $M^{\prime}$ is $p$-nilpotent, by the inductive hypothesis, implies that $M^{\prime}$ is a $p$-group because $O_{p^{\prime}}(G)=1$. Then $P_{1}$ is characteristic in $M$ and since $M \triangleleft G$, we have $P_{1} \triangleleft G$. If $P \triangleleft G$, then $G / P$ is abelian and since all subgroups $H$ of $P$ with $|H|=|D|$ are $S$-permutable in $G$, we have that $G$ is supersolvable by ([14], Theorem 1.3) and so $G^{\prime}$ is nilpotent; in particular $G^{\prime}$ is $p$-nilpotent. Thus we may assume that $P \Varangle G$ and $P_{1}=F(G)$ the Fitting subgroup of $G$ (recall that $O_{p^{\prime}}(G)=1$ and that $F(G)=<O_{p}(G)$ for all $p$ divides $\left.|G|>\right)$. Consider the subgroup $\Phi\left(P_{1}\right)$ and assume that $\Phi\left(P_{1}\right) \neq 1$. Hence if $\left|\Phi\left(P_{1}\right)\right|<|D|$, then $\left(G / \Phi\left(P_{1}\right)\right)^{\prime}$ is $p$-nilpotent by induction on $|G|$; in particular $G^{\prime}$ is $p$-nilpotent. So assume that $\left|\Phi\left(P_{1}\right)\right| \geq|D|$. If $\left|\Phi\left(P_{1}\right)\right|=|D|$, then $P / \Phi\left(P_{1}\right)$ is not cyclic. Let $L / \Phi\left(P_{1}\right)$ be a proper subgroup of $P / \Phi\left(P_{1}\right)$ such that $\left|L / \Phi\left(P_{1}\right)\right|=p(L$ is not cyclic; otherwise $\Phi\left(P_{1}\right)$ is cyclic and this implies that there exists $L_{1} \leq \Phi\left(P_{1}\right)$ such that $L_{1} \triangleleft G$; in particular $G / C_{G}\left(L_{1}\right)$ is isomorphic to a subgroup of $A u t\left(L_{1}\right)$ and so $G^{\prime} \leq$ $C_{G}\left(L_{1}\right)$ and we conclude then that $G^{\prime}$ is $p$-nilpotent). As $\left|L / \Phi\left(P_{1}\right)\right|=p$, then there exists a maximal subgroup $L_{1}$ of $L$ such that $\left|L_{1}\right|=\left|\Phi\left(P_{1}\right)\right|=|D|$ and $L_{1} \neq \Phi\left(P_{1}\right)$. But $L_{1} \Phi\left(P_{1}\right)$ is $S$-permutable in $G$, then $L_{1} \Phi\left(P_{1}\right) / \Phi\left(P_{1}\right)=L / \Phi\left(P_{1}\right)$ is $S$-permutable in $G / \Phi\left(P_{1}\right)$. By Theorem 2.1, $\left(G / \Phi\left(P_{1}\right)\right)^{\prime}=G^{\prime} \Phi\left(P_{1}\right) / \Phi\left(P_{1}\right)$ is $p$-nilpotent and so $G^{\prime}$ is $p$-nilpotent. Thus we may assume that $\Phi\left(P_{1}\right)=1$ and $P_{1}$ is elementary abelian. Since all subgroups $H$ of $P_{1}$ with $|H|=|D|$ are normal in $M$, we have by ([1], Lemma 2.9) that all subgroups of $P_{1}$ of order $p$ are normal in $M$. So $P_{1} \cap Z(P) \neq 1$. Let $L \leq P_{1} \cap Z(P)$ such that $|L|=p$. Then $L \triangleleft G$ and since $G / C_{G}(L)$ is isomorphic to a subgroup of $A u t(L)$, we have that $G^{\prime} \leq$ $C_{G}(L)$, in particular $G^{\prime} L / L$ is $p$-nilpotent and so $G^{\prime}$ is $p$-nilpotent. This completes the proof of the theorem.

\section{References}

[1] M. Asaad and A. A. Heliel, On $S$-quasinormally embedded subgroups of finite groups, JPAA 165(2001) 129-135.

[2] A. Ballester-Bolinches and X. Guo, On complemented subgroups of finite groups, Arch. Math. 72(1999) 161-166.

[3] A. Ballester-Bolinches, Y. Wang and X. Guo (2000), C-supplemented subgroups of finite groups, Glasgow Math. J. 42(2000) 383-389.

[4] J. Buckely, Finite groups whose minimal subgroups are normal, Math. Z. 116(1970) $15-17$.

[5] D. Gorenstein, Finite Groups, American Mathematical Society, 1980.

[6] P. Hall, A characteristic property of solvable groups, J. London Math. Soc. 12(1937) 198-200.

[7] P. Hall, Complemented Groups, J. London Math. Soc. 12(1937) 201-204. 
[8] A. A. Heliel, A note on $c$-supplemented subgroups of finite groups, Comm. Algebra 42(2014) 1650-1656.

[9] R. Hijazi, A note on solvability of finite groups, Journal of Advances in Mathematics 10(2015) 3639-3642.

[10] B. Huppert, Endliche Gruppen I, Springer, Berlin-New York, 1979.

[11] O. H. Kegel, Sylow-Gruppen und Subnormalteiler endlicher Gruppen, Math. Z. 78(1962) 205-221.

[12] J. Li, W. Shi, G. Chen and D. Yu, New characterization of solubility of finite groups, Italian J. Pure Appl. Math. 33(2014) 377-382.

[13] P. Schmid, Subgroups permutable with all Sylow subgroups, J. Algebra, 207 (1998) 285-293.

[14] A. N. Skiba, On weakly $S$-permutable subgroups of finite groups, J. Algebra, 315(2007) 192-209.

[15] S. Srinivasan, Two sufficient conditions for supersolvability of finite groups, Israel J. Math. 35(1980) 210-214. 